\title{
Balloon-Borne Gamma-Ray Polarimeter (PoGO) to study Black Holes, Pulsars, and AGN Jets: Design and Calibration
}

\author{
Zachary Apte \\ Office of Science, SULI Program \\ Hampshire College \\ Stanford Linear Accelerator Center \\ Menlo Park, California
}

July 11, 2005

Prepared in partial fulfillment of the requirements of the Office of Science, U.S. Department of Energy Science Undergraduate Laboratory Internship (SULI) Program under the direction of Dr. Tsunefumi Mizuno, Dr. Tsuneyoshi Kamae, and Dr. Grzegorz Madejski in the Astro Gamma group of the Stanford Linear Accelerator Center.

Participant:

Signature

Research Advisor:

Signature 


\section{Table of Contents}

Abstract iii

$\begin{array}{ll}\text { Introduction } & 1\end{array}$

Flight Instrument 3

Materials and Methods $\quad 4$

$\begin{array}{lr}\text { Results } & 6\end{array}$

$\begin{array}{ll}\text { Discussion and Conclusions } & 7\end{array}$

$\begin{array}{ll}\text { Acknowledgements } & 8\end{array}$

$\begin{array}{lr}\text { References } & 8\end{array}$

$\begin{array}{ll}\text { Tables } & 10\end{array}$

$\begin{array}{ll}\text { Figures } & 10\end{array}$ 


\begin{abstract}
Gamma-Ray Polarimeter to study Black Holes, Pulsars, and AGN Jets: Design and Calibration. ZACHARY APTE (Hampshire College, Amherst, MA 01002) Tsuneyoshi Kamae (Stanford Linear Accelerator Center, Menlo Park, CA 94025)
\end{abstract}

Polarization measurements at X-ray and gamma-ray energies can provide crucial information on the emission region around massive compact objects such as black holes and neutron stars. The Polarized Gamma-ray Observer (PoGO) is a new balloon-borne instrument designed to measure polarization from such astrophysical objects in the $30-100 \mathrm{keV}$ range, under development by an international collaboration with members from United States, Japan, Sweden and France. The PoGO instrument has been designed by the collaboration and several versions of prototype models have been built at SLAC. The purpose of this experiment is to test the latest prototype model with a radioactive gamma-ray source. For this, we have to polarize gamma-rays in a laboratory environment. Unpolarized gamma-rays from Am241 (59.5 keV) were Compton scattered at around 90 degrees for this purpose. Computer simulation of the scattering process in the setup predicts a $86 \%$ polarization. The polarized beam was then used to irradiate the prototype PoGO detector. The data taken in this experiment showed a clear polarization signal, with a measured azimuthal modulation factor of $0.35 \pm 0.02$. The measured modulation is in very close agreement with the value expected from a previous beam test study of a polarized gamma-ray beam at the Argonne National Laboratories Advanced Photon Source. This experiment has demonstrated that the PoGO instrument (or any other polarimeter in the energy range) can be tested in a libratory with a simple setup to a similar accuracy. 


\section{INTRODUCTION}

Accreting black holes, isolated pulsars, and jet-dominated active galactic nuclei (AGN) are among the many exotic objects of great astronomical interest which can emit polarized xrays and gamma rays; measurements of the polarization in the Compton scattering energy range (30-100keV) can reveal important information about the conditions in and around these mysterious objects. The Polarized Gamma-ray Observer (PoGO) project is an international effort to send a very sensitive instrument to the top of the atmosphere (overburden of $3 \mathrm{~g} / \mathrm{cm}^{2}$ ) to measure polarization of these sources.

Polarization of astrophysical gamma-rays arises thorough a variety of well-understood processes. Synchrotron radiation arises due to high energy electrons orbiting in an ordered magnetic field [3]. All synchrotron radiation is polarized perpendicular to the magnetic field revealing information about the orientation of the magnetic field around the source. Compton scattering of unpolarized photons from the accretion disk or surrounding clouds around a black hole or an active galactic nucleus by high energy electrons can also produce polarized gamma-

rays[9]. The absorption cross-section of photons traveling through a strong magnetic field $\left(>10^{12}\right.$ Gauss) depends on the polarization direction making it possible to measure the strong-field near the surface of a neutron star [1,2]. In all of these cases, the orientation of the polarization plane (the plane on which the electric field vector lies) depends on the orientations of the magnetic field or the accretion disk; therefore polarization measurement is a powerful probe of the emission mechanism and the source geometry[9]. Due to the lack of sensitivity, there has been no measurement of polarization in X-ray and gamma-ray bands except for that on Crab Nebula in early 1970s[6]. 
Despite the importance of the x-ray and gamma-ray polarization measurement, it has only been measured only once by the OSO-8 satellite which studied the Crab Nebula at 2.6 and 5.2 $\mathrm{keV}$. We note that the RHESSI satellite reported a polarization measurement using Bragg diffraction [5,6,7]2 years ago for a gamma-ray burst [8] but the validity of the measurement has been severely challenged.

To examine the PoGO flight instrument's capability, a simplified prototype detector array was recently tested at the Advanced Photon Source(APS) at Argonne National Laboratories[9]. The detector array consisted of seven hexagonally configured fast plastic scintillators coupled with PMTs [figure 2] and was irradiated by polarized photon beams at 60, 73 and $83 \mathrm{keV}$. The data showed an azimuth-angle modulation factor of $0.42 \pm .01$. Computer simulations of this experiment using a modified Geant4 simulation package showed a modulation factor of 0.488 \pm .006 for a fully polarized $73 \mathrm{keV}$ photon beam[9].

The experiment reported here will focus on repeating the capability tests previously conducted at APS in the laboratory using a simple laboratory setup which utilizes the radioactive source Americium 241 (59.5 keV): if we obtain a similar result in this experiment, it will become a very powerful verification method in the laboratory during development of the PoGO flight instrument.

To create a polarized beam, Compton photons scattered around 90 degrees are selected in a double scattering setup[figure 3]. Polarization is a function of scattering angle and reaches 100\% at 90 degrees. The Klein-Nishina cross section (1) takes the following formula

$$
\frac{d \sigma}{d \Omega}=\frac{1}{2} r_{0}^{2} \frac{k^{2}}{k_{0}^{2}}\left[\frac{k}{k_{0}}+\frac{k_{0}}{k}-2 \sin ^{2} \theta \cos ^{2} \phi\right]
$$

where $\theta$ is the scattering angle, or angle between the incident and the scattered gamma-rays, $\&$ the angle between the electric field plane and he scattered gamma ray 
direction. The notation is defined as in Figure 2 . There, one completely linearly polarized photon is scattered by a free electron at point $\mathrm{O}$. The momentum vector of incident and scattered photon are $k_{0}$ and $k$, respectively, and the unit vector along the polarization vector before the scattering is $e_{0} . \theta$ and $\varphi$ are the polar and azimuth angle of the scattering. Here, $k_{0}$ and $e_{0}$ are assumed to be along z-axis and $\mathrm{x}$-axis, respectively.

Because we are scattering at 90 degrees, the value for $\theta$ is 90 degrees(from horizontal to vertically down), making sine squared go to 1 . Then after that photon scatters off the central scintillator (back to horizontal) the cosine squared value goes from zero to one, creating a angular modulation in the differential cross-section.

Another important Compton equation is (2) which describes the energy of the scattered photon (E') as a function of the scattering angle $\theta$ and the initial energy E.

$$
E^{\prime}=\frac{E}{1+\frac{E}{M_{e} c^{2}}(1-\cos \theta)}
$$

\section{FLIGHT INSTRUMENT}

To carry out polerization measurements we are constructing a new balloon-borne instrument, the Polarized Gamma-ray Observer (PoGO ) $[9,10]$ which employs coincident detection of Compton scattering and photo-absorption to measure polarization in the $30-100 \mathrm{keV}$ range [9]. The flight instrument will carry 217 bundled well-type phoswich counters with PMTs (Photo Multiplier Tubes). Each counter will consist of three types of scintillation material: one Bismuth germinate crystal (BGO) and two types of plastic scintillators, one with a fast response (fast plastic) and another with a slow response (slow plastic). The 217 phoswich counters will be surrounded by the side BGO scintillators [Figure 1]. 
The instrument employs a well-type phoswich active shielding design to produce high quality data (high signal-to-noise factor) from low flux sources[11]: each type of scintillation material has a very different temporal response to incident $\mathrm{x}$-rays; making discrimination between the location of a scintillation signal possible. The BGO scintillators are used to block background x-rays from the bottom and sides: any event detected in coincidence with an event in the BGO scintillators it is discarded. The slow plastic scintillators $(60 \mathrm{~cm}$ long and hollow ) with tin and lead foils wrapped around them define the field of view of the instrument to about two degrees by two degrees. The only $\mathrm{x}$-rays which will produce a signal at a fast scintillator but not at slow plastic will be recorded as "valid" events[Figure 1].

The fast plastic scintillators are the main detector of the instrument. X-rays that enter a fast scintillator, Compton-scatter into another fast scintillator, and are photo-absorbed there will make a valid event. From the coincident set of fast scintillators, the path of the x-ray can be reconstructed and the direction of the Compton scattering determined. Because the asymmetry (modulation) in azimuth angle of Compton scattering is directly related to the polarization of the x-rays, the polarization (magnitude and orientation) of the x-rays can be determined.

\section{MATERIALS AND METHODS}

To create a mono-energetic, collimated, polarized gamma ray source a strong Americium 241 source was placed inside a lead container with a $5 \mathrm{~mm}$ wide and $39.5 \mathrm{~mm}$ long hole in it. The small hole leads to a fast scintillator, also contained in the lead, with another hole (5mm by $50 \mathrm{~mm}$ ) at 90 degrees to the first, leading out of the container. This setup should only allow events Compton scattered at 90 degrees out, creating a mono-energetic beam (53.3 keV, post 
scattered energy see eq. 2) that has a high degree of polarization and is highly collimated[Figure 3].

The PoGO Prototype Detector was arranged as an array of 7 hexagonal plastic SaintGobain BC-404 scintillators, each $2.68 \mathrm{~cm}$ wide and $20 \mathrm{~cm}$ long, as shown in Figure 4, 5 (which includes the numbering scheme). Each scintillator was glued directly to a Hamamatsu R580 PMT (3.4 cm photocathode diameter, larger than that of PMTs used for PoGO flight). The center scintillator acted as a Compton scattering target and the outer six scintillators, separated from the center scintillator by $2.2 \mathrm{~cm}$, detected the scattered photons. In the final PoGO instrument the hexagonal detector units will be tightly bundled together in a close-packed hexagonal array figure [1][12]. However, photons scattered in one detector are likely to pass through to its nearest neighbors before being photo-absorbed. Thus, the prototype array approximates a region of the final PoGO instrument[9].

The array was mounted on a rotation stage, as shown in Figure 5, to allow measurement of the modulation factor by rotating about the center scintillator aligned to the incident photon beam. To measure the detector response to relative to the polarization plane, the instrument was rotated in 30 degree steps covering the azimuthal angle range of 0-150 degrees. Each run took about 60 hours. Energy measured by the fast scintillator was calibrated by irradiating each detector am 241[figure 11]. The energy resolution (full width at half maximum) at $59.5 \mathrm{keV}$ spanned the range of $31 \%$ to $39 \%$ and were assumed to scale with the inverse square root of energy [9]. Coincidence triggering was used to gate our data acquisition system as shown in figure 12 .

To eliminate background events which were not removed by hardware triggering, several different data selection criteria were used finally producing a clean data set of approximately 20 
Compton events per hour. To remove background from cosmic-rays an initial set of selection criteria were used to analyze the waveform output from the PMTs, only events which had the temporal response corresponding to a scintillation event were selected. Those data were then converted from volts to $\mathrm{keV}$ and further event selection criteria were applied to select only valid Compton events (photons scattered off the central scintillator and absorbed by a peripheral one): requiring that exactly 2 PMTs had an energy greater then $3 \mathrm{keV}$, and that the total energy in all the PMTs must be between 39.5 and $79.5 \mathrm{keV}(59.5 \pm 20)$. The events in each of the peripheral scintillators which made it through all the criteria were then summed to give the number of events in each peripheral scintillator.

A modified Geant4 toolkit [13] was used to find a modulation factor for a Monte Carlo simulation of the prototype configuration being irradiated with polarized $53.3 \mathrm{keV}$ photons. The simulation didn't take into account the Gaussian energy response of the scintillation materials so a Gaussian pseudo-random number generator was used to apply a Gaussian energy distribution.

We normalized the number of events in each scintillator to the integration time and then fit the result to a sinusoidal curve and obtained a modulation factor $\left(M_{p}\right)$ from the maximum $(R \max )$ and minimum $(R \min )$ rates measured as a function of azimuthal angle by [Figure 8]:

$$
M_{p}=\frac{R_{\max }-R_{\min }}{R_{\max }+R_{\min }}
$$

\section{RESULTS}

Using the aforementioned software event selection we obtained a clean data set with around 20 recorded Compton events per hour. A plot of the energy distribution of events recorded in PMT8, the fast scintillator in the lead brick used to create the polarization (triggered with PMT4, the central scintillator), shows the expected Poissan energy distribution around 6.2 
$\mathrm{keV}$ for Compton scattering of $59.5 \mathrm{keV}$ photons at 90 degrees [Figure 7]. The event selection used can be clearly seen superimposed over the plot of energy deposited in the central scintillator versus the total deposited energy[Figure 8]. There are several structures visible in Figure 8 that we used event selection to exclude. The clear line along the bottom of the cluster of data points is composed of events which only include PMT4; the higher energy ones are photo-absorption and the lower energy ones are escaped Compton events. Table 1 is a table of the all the modulation factors acquired over the course of this experiment. The modulation factor acquired over this experiment is $86 \%$ of the one acquired one at the Argonne beam test[9] and $74 \%$ of the value indicated by the simulation[Figure 9].

\section{DISCUSSION AND CONCLUSION}

We succeeded in measuring polarization in the laboratory which was the motivation of this paper has implications into the PoGO project as a whole. The characterization of this method is important because for development of the PoGO flight instrument because instrument development demands a much more flexible schedule of testing than available at a source such as APS. Computer simulation using the Geant 4 analyses package indicates double scattering events will lead to an $86 \%$ polarized beam [Figure 10]. Because we measured the polarization to be about $86 \%$ of the one measured at the Argonne APS, and simulations indicate that double scattering events inside the scattering scintillator lead to an $86 \%$ polarization, our data seem to validate this method.

Because of the angular width of the central scintillator and the angular size of the holes in the lead brick, the scattering angle is not truly 90 degrees but is the whole range of 80-100 degrees. The maximum and minimum normalized differential cross-sections for this situation 
range from .96 to 1 and 0 to .04 respectively, leading to $\pm 4 \%$ difference in polarization. This is for a scintillator perfectly aligned with the beam; if the scintillator was not perfectly aligned the overall error could increase to $\pm 5 \%$.

Our result normalized to the Argonne beam test result $(0.36 / 0.42=0.86)$ matches closely with the simulation's value for polarization (86\%), confirming the validity of this method as a development tool for the PoGO flight instrument. 


\section{ACKNOWLEDGEMENTS}

I would like to thank the U.S. Department of Energy for giving me the opportunity to participate in the SULI program, the experience had been invaluable. Dr. Tsunifume Mizuno deserves special thanks for working closely with me on this project. Additionally, many thanks to both Dr. Tsuneyoshi Kamae, and Dr. Grzegorz Madejski who helped extensively with the editing of this paper.

\section{REFERENCES}

[1] A. K. Harding, Physics in Strong Magnetic Fields in Science, vol. 251 pp 1033 March 1991

[2] A. K. Harding, The Physics of Gamma-ray Bursts Physics Reports vol. 206 pp. 327-291 February 1991

[3] G.B. Rybicki, A.P. Lightman, Radiative Processes in Astrophysics, New York: Wiley, 1979

[4] K. Makishima et al., A. S. P Conf. Proc. 251, ed. H. Inoue and H. Kunieda, Astr. Soc. of the Pacific (2001) 564.

[5] M. C. Weisskopf, et. Al, Measurement of the X-ray Polarization of the Crab Nebula, Astrophysical Journal, vol. 208, pp L125-L128, June 1976.

[6] M. C. Weisskopf, et. Al, A Precision Measurement of the X-ray Polarization of the Crab Nebula without Pulsar Contamination, Astrophysical Journal vol. 220 pp. L117-L121 March 1978

[7] E. H. Silver, et. Al, Search for X-ray polarization in the Crab Pulsar, Astrophysical Journal, volume 225 pp. 221-225, October 1978.

[8] W. Coburn, S. Boggs, Polarization of the prompt -ray emission from the -ray burst of 6 December 2002 Nature, vol. 423 pp 415-417 May 2003

[9] T. Mizuno, et. Al. Beam Test of a Prototype Detector Array for the Pogo Astronomical Hard X-Ray/Soft Gamma Ray Polarimeter, Nuc. Inst. Met'd. phy res 540 (2005) 158-168

[10] V. Andersson, et. Al Large-Area Balloon-Borne Polarized Gamma Ray Observer (PoGO) $22^{\text {nd }}$ Texas Symposium, Stanford, December 13-17 2004 
[11] T Kamae, et. Al., Well-Type Phoswich Counter for Low-Flux X-Ray/ $\gamma$ Detection, IEEE Trans. Nucl Sci., vol. 40, no. 2, pp. 204-207, Apr. 1993

[12] P. Chen et al., in Proc. IEEE Nuclear Science Symp., Portland, Oregon, October 2003

[13] S. Agostinelli et Al, Geant4 - a simulation toolkit, Nucl. Instr. and Meth., vol. 506 pp. 250 - 303 August 2003 


\section{TABLES}

Table 1

\begin{tabular}{|c|c|c|c|c|c|c|}
\hline \multicolumn{7}{|c|}{ Results over different runs } \\
\hline Data Source & $\begin{array}{l}\text { offset from initial } \\
\text { position(degrees) }\end{array}$ & $\begin{array}{l}\text { Modulation } \\
\text { factor }\end{array}$ & $\begin{array}{l}\text { total } \\
\text { counts }\end{array}$ & $\begin{array}{l}\text { time period } \\
\text { (hr) }\end{array}$ & $\begin{array}{l}\text { compton } \\
\text { events/hr }\end{array}$ & \\
\hline AM241 & 0 & $0.39 \pm 0.04$ & 1343 & 65.5 & & 20.5 \\
\hline AM241 & 30 & $0.33 \pm 0.038$ & 1192 & 63 & & 18.9 \\
\hline AM241 & 60 & $0.32 \pm .042$ & 1289 & 66 & & 19.5 \\
\hline AM241 & 90 & $0.35 \pm 0.036$ & 1158 & 60 & & 19.3 \\
\hline AM241 & 120 & $0.36 \pm 0.044$ & 1141 & 58.5 & & 19.5 \\
\hline AM241 & 150 & $0.39 \pm 0.036$ & 1113 & 60 & & 18.6 \\
\hline Average & NA & $.036 \pm .002$ & 7236 & 373 & & 19.4 \\
\hline SIMULATION & 30 & $0.488 \pm 0.001$ & 13959 & NA & NA & \\
\hline
\end{tabular}

\section{FIGURES}

Figure 1. Conceptual design of PoGO. It consists of an array of well-type phoswich detector units, each made of a fast plastic scintillator, a slow plastic scintillator tube, a thin high-Z metal foil and a bottom BGO. A set of side anti-coincidence detectors made of BGO surrounds the array of phoswich units. In the figure, representative passages of gamma-rays are shown with energy deposition marked by circles. The trigger scheme accepts only the ones marked as "Fully contained gamma-ray”.

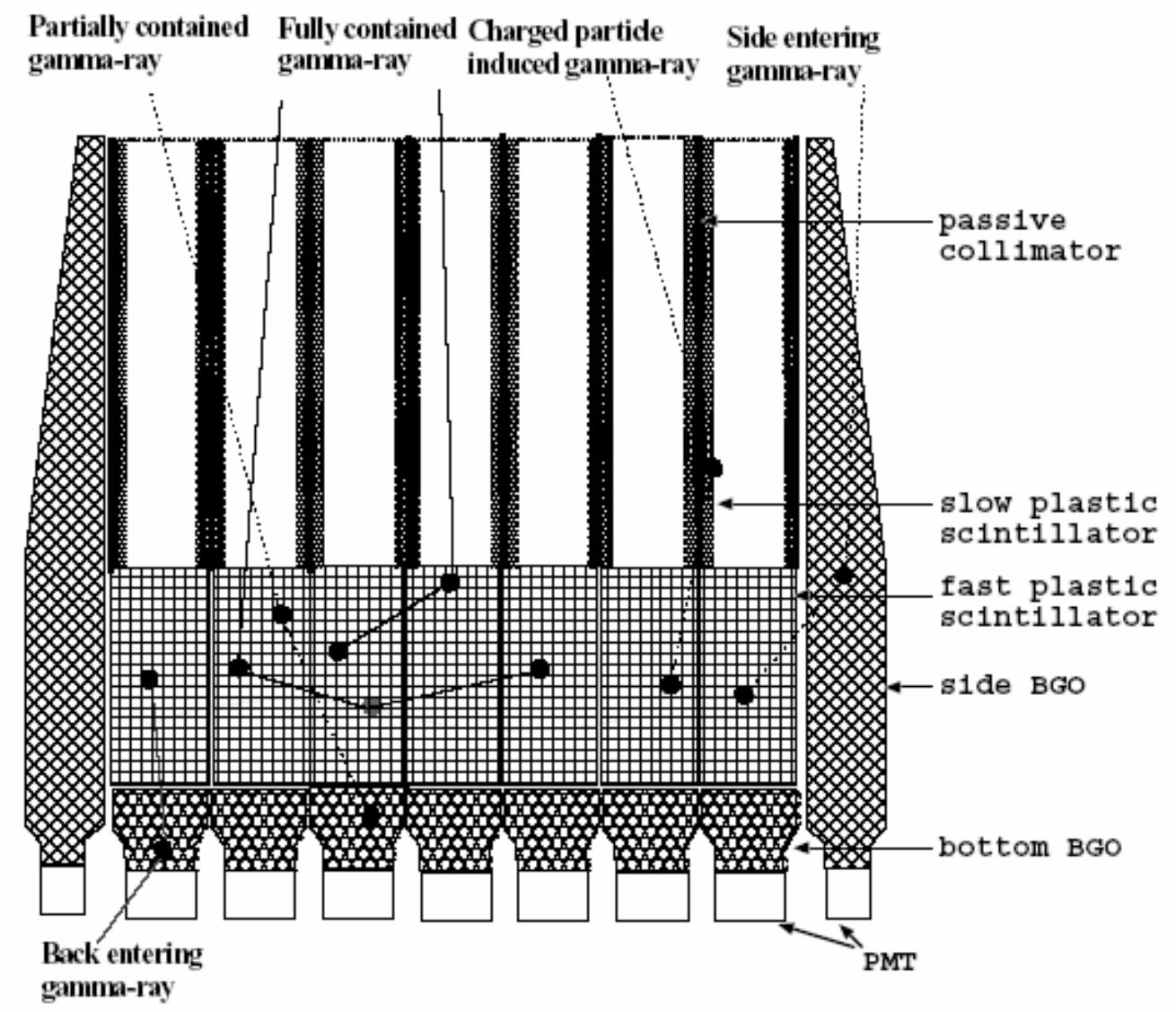


Figure 2. A photon scattering at point $\mathrm{O}$. The momentum vector $\left(k_{0}\right)$ and the polarization vector $\left(e_{0}\right)$ of an incident photon are along $\mathrm{z}$ - and $\mathrm{x}$-axis, respectively. $\theta$ and $\varphi$ are the scattering polar and azimuth angle. Plane $S$ is constructed by $e_{0}$ and $\_k$, the momentum vector after the scattering. $e$ is in plane $\mathrm{S}$.
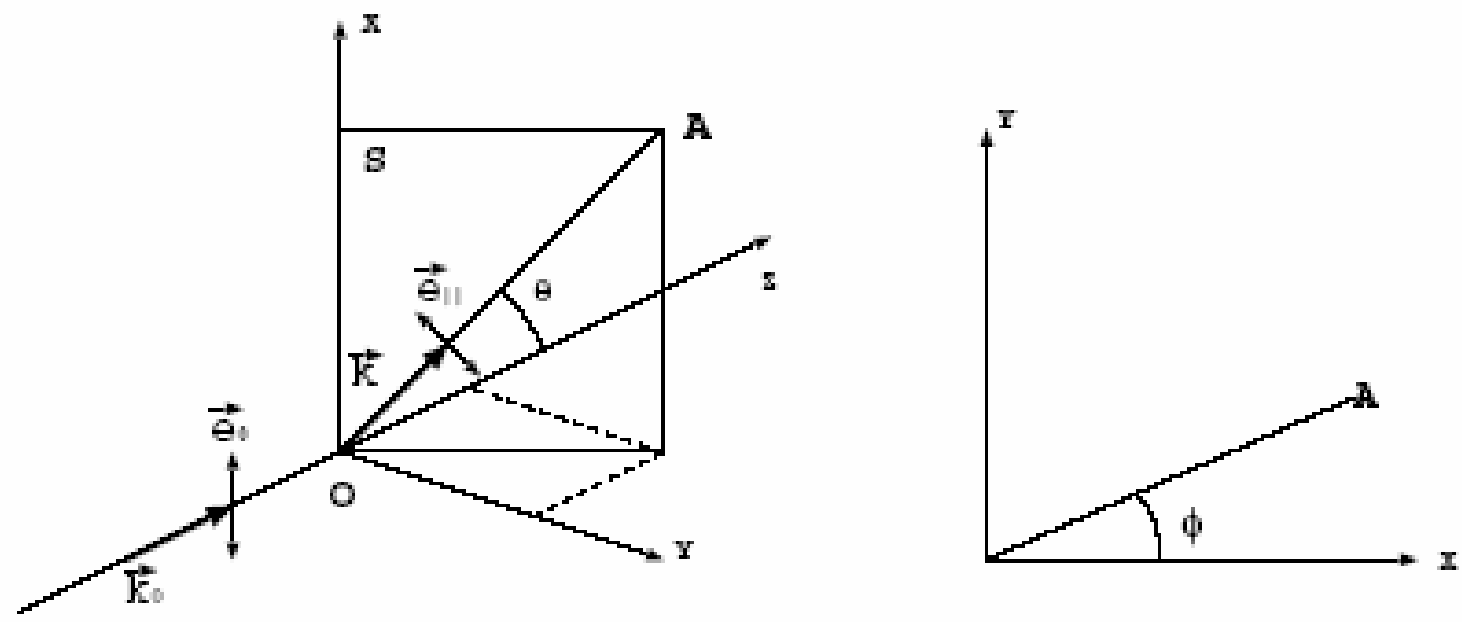

Figure 3. A Simplified drawing of the scattering apparatus. The large green block represents the lead block that the Americium (blue) and PMT8 (red) are embedded in. The white tubes represent the holes in the lead block. The dashed red line represents a possible gamma ray path. The jagged shape represents the Compton event.

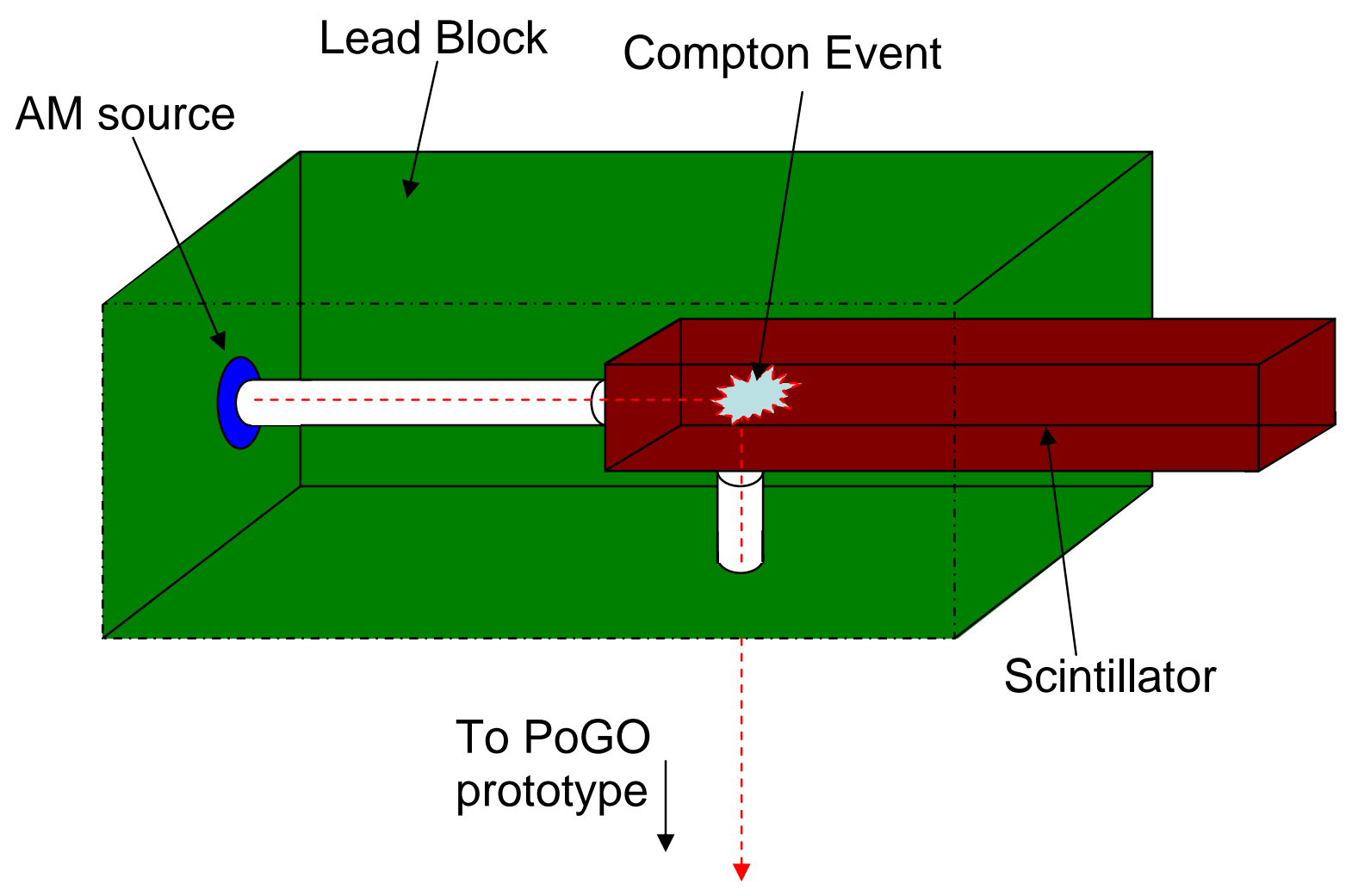


Figure 4. The layout and numbering scheme of scintillators viewed from the beam origin. Detector rotation angle is defined to be $0^{\circ}$ when scintillators channels 3,4 and 5 are along the horizontal (x-axis), and to be $30^{\circ}$ when channels 1,4 and 7 are along the vertical (y-axis).

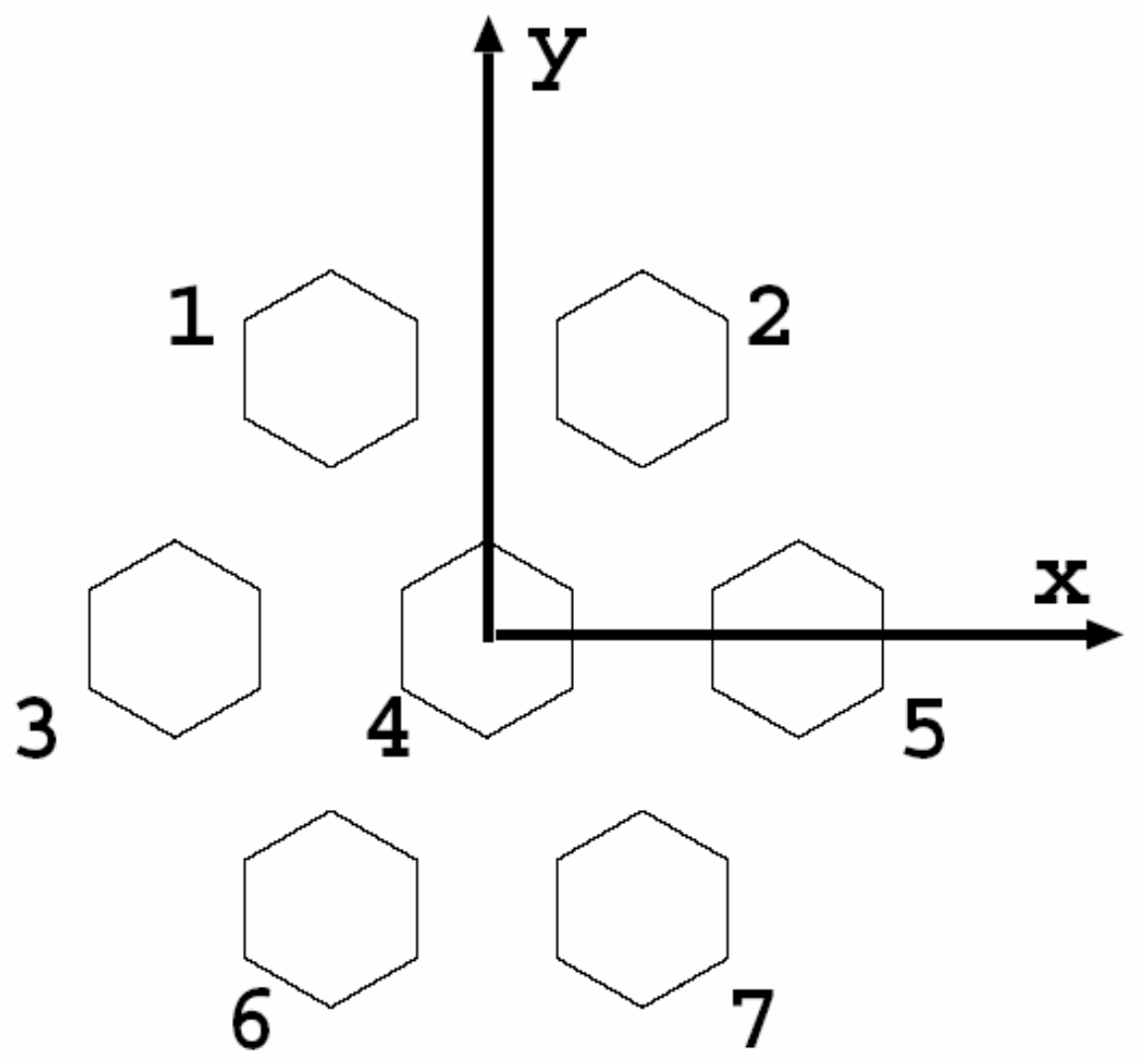

Figure 5. The rotation frame containing the 7 PMTs.

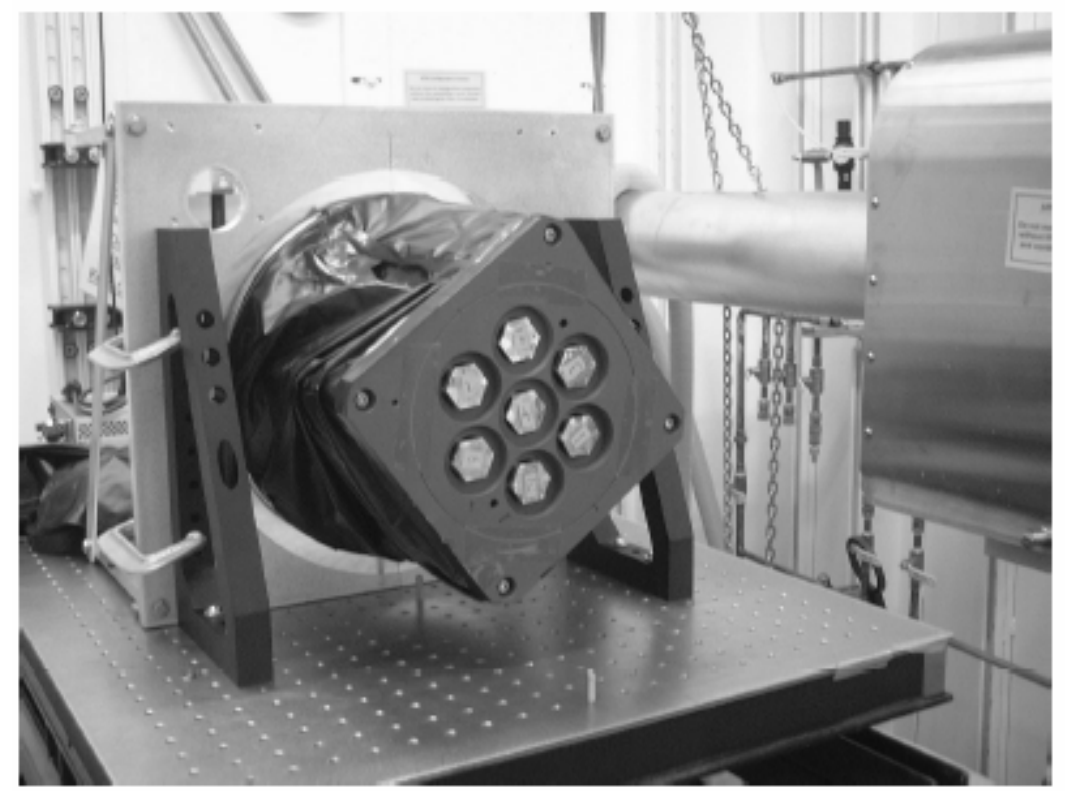


Figure 7. This plot shows Poissan distribution of energy for 90 degree Compton events in PMT8, the polarization apparatus ( $\sim 6.2 \mathrm{keV}$ absorption). As well the higher peak for the double scattering can be seen.

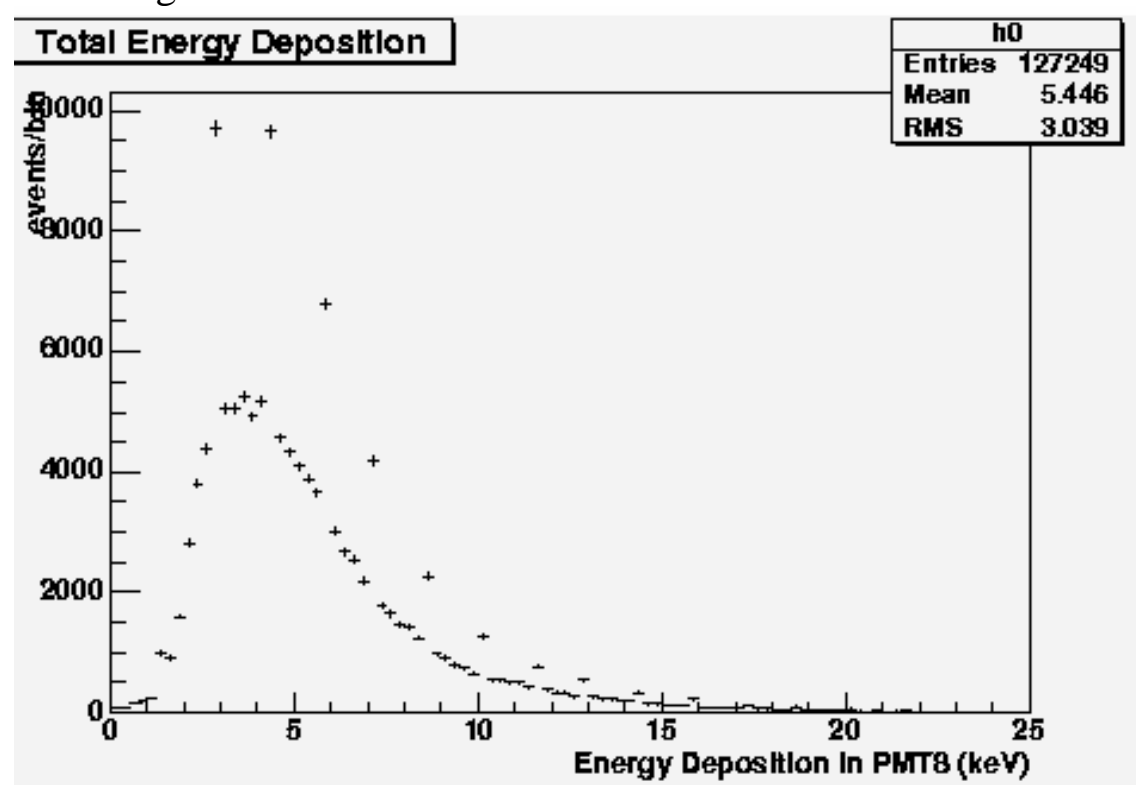

Figure 8. Relation of deposit energy in the central scintillator and total energy deposition for $59.5 \mathrm{keV}$ run at 0-degree rotation. Event selection criteria used in data analysis are also shown by dotted lines. (see text)

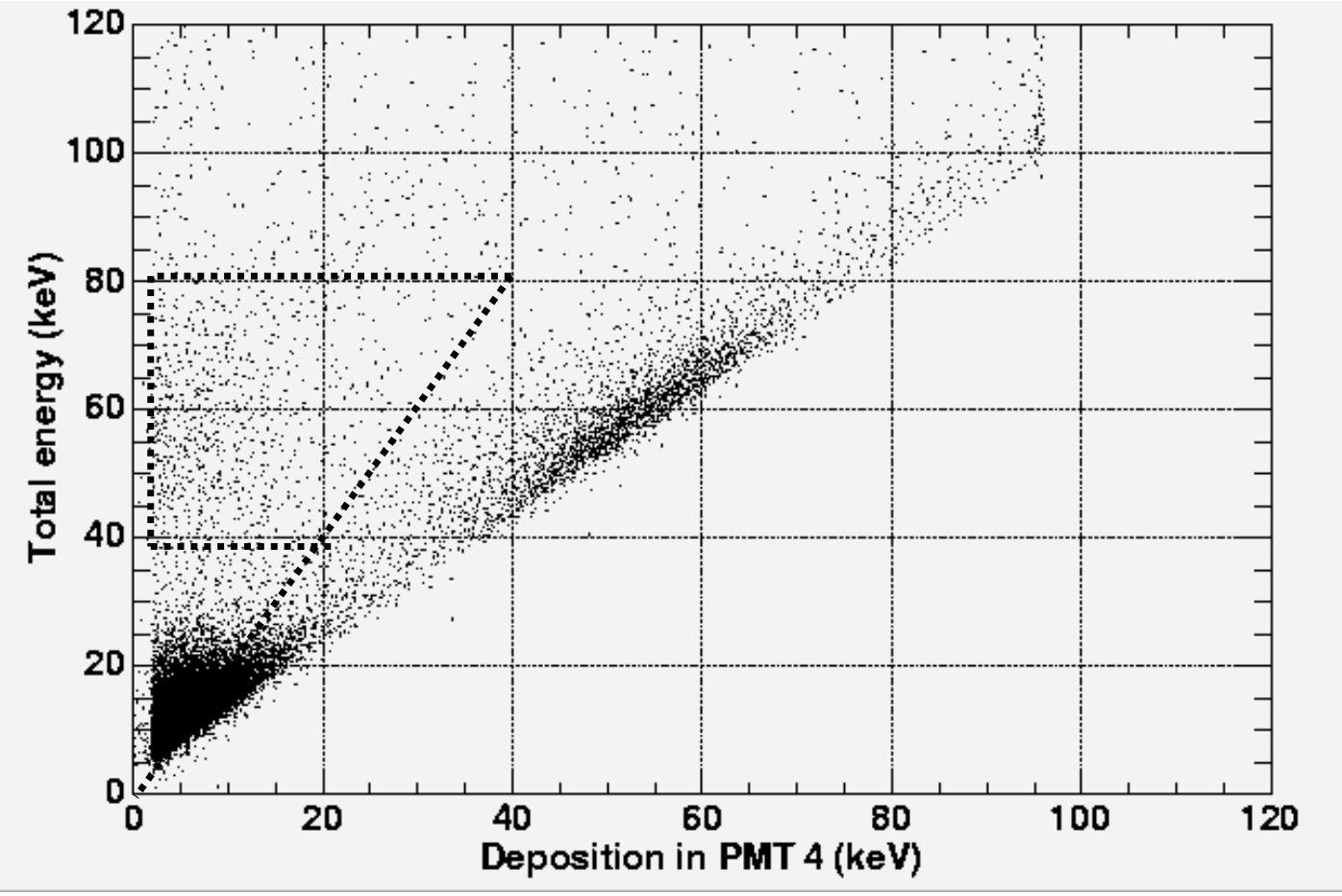


Figure 9. Simulation of polarization from lab setup using taking double scattering into account leads to an $86 \%$ polarization.

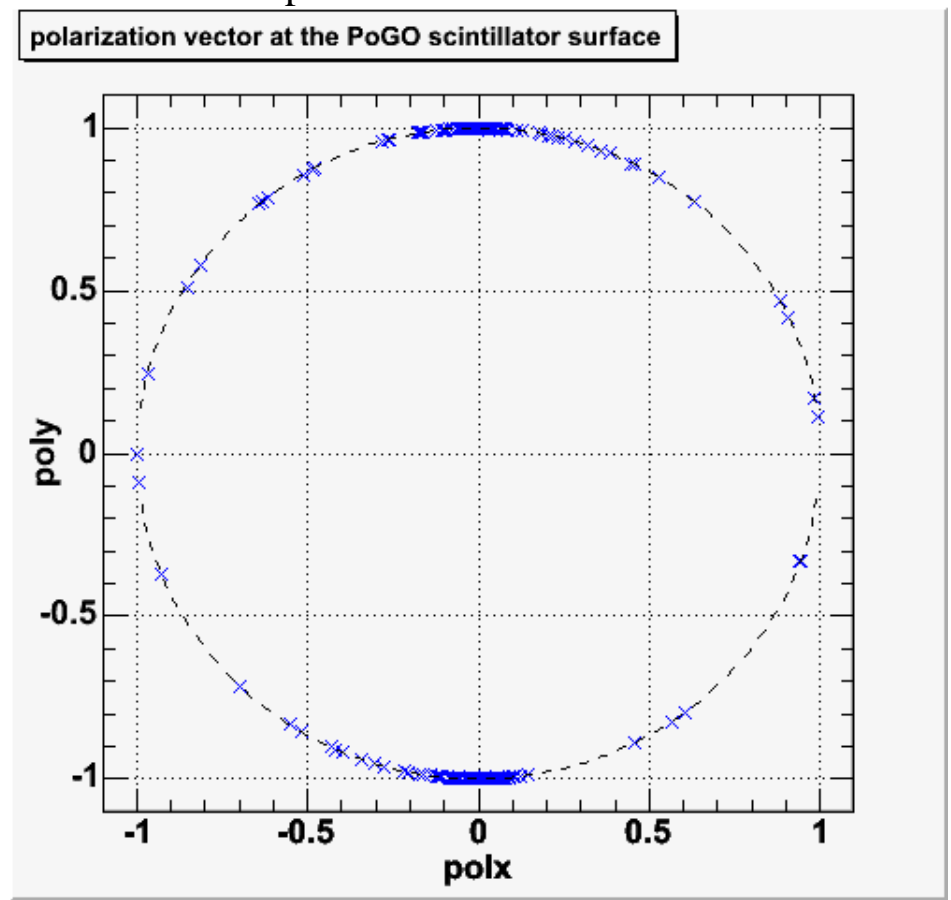

Figure 10. Modulation curves for PMTs, and overlaid with the simulation curve.

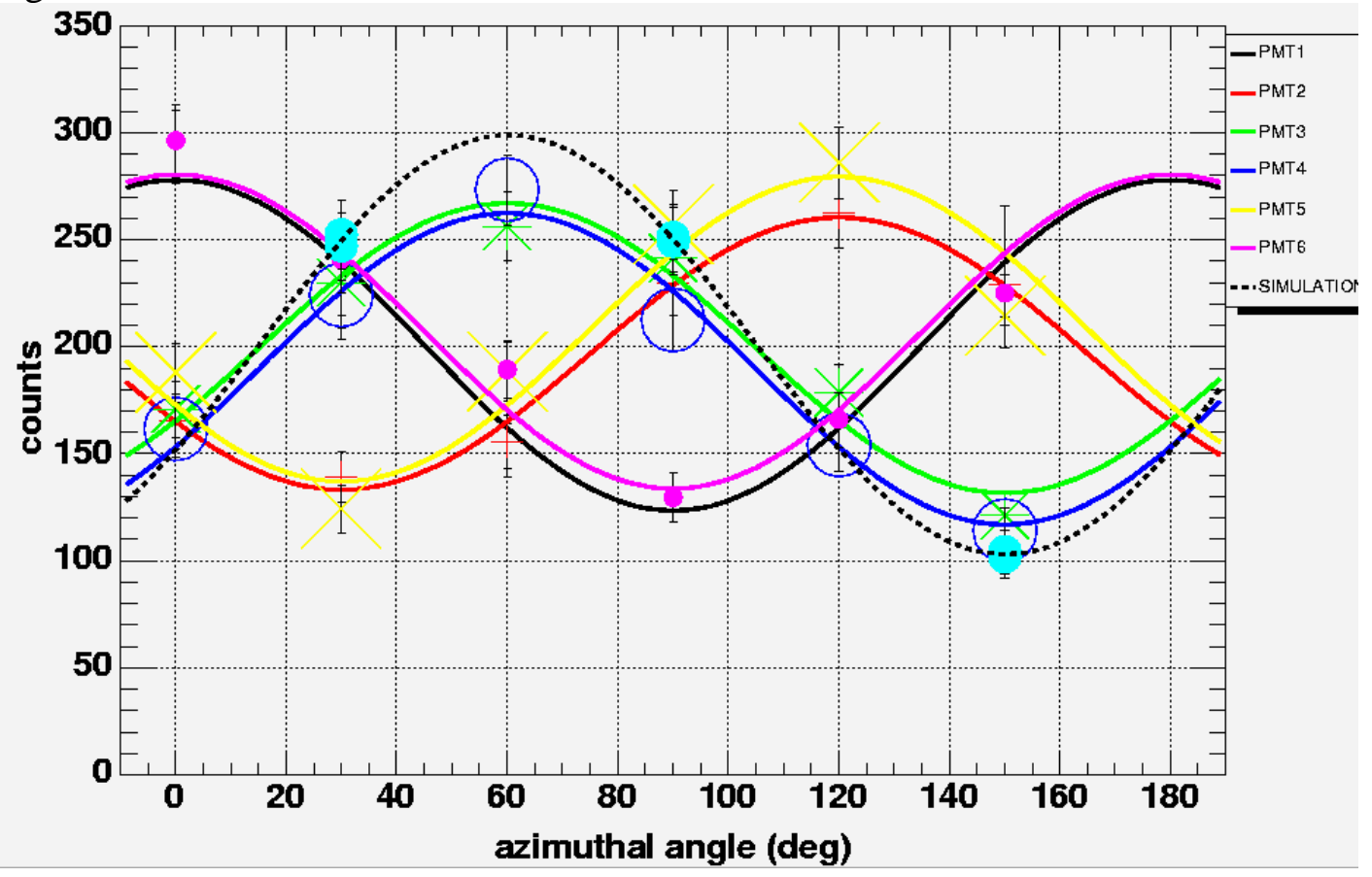


Figure 11. Calibration curve of a PMT scintillator pair. Obtained by irradiating the PMT with $59.5 \mathrm{keV}$ photons.

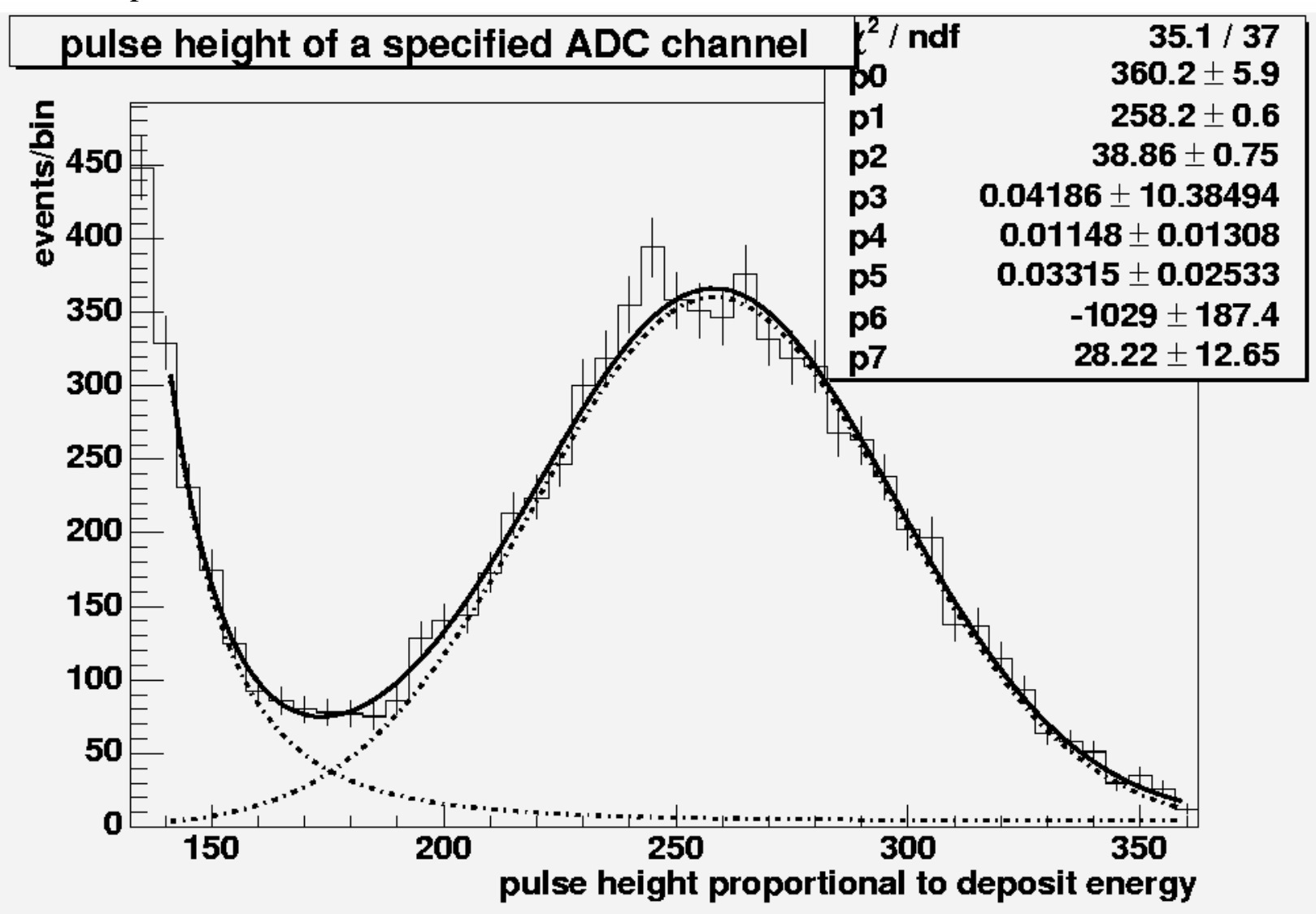

Figure 12. The data flow for our experiment.

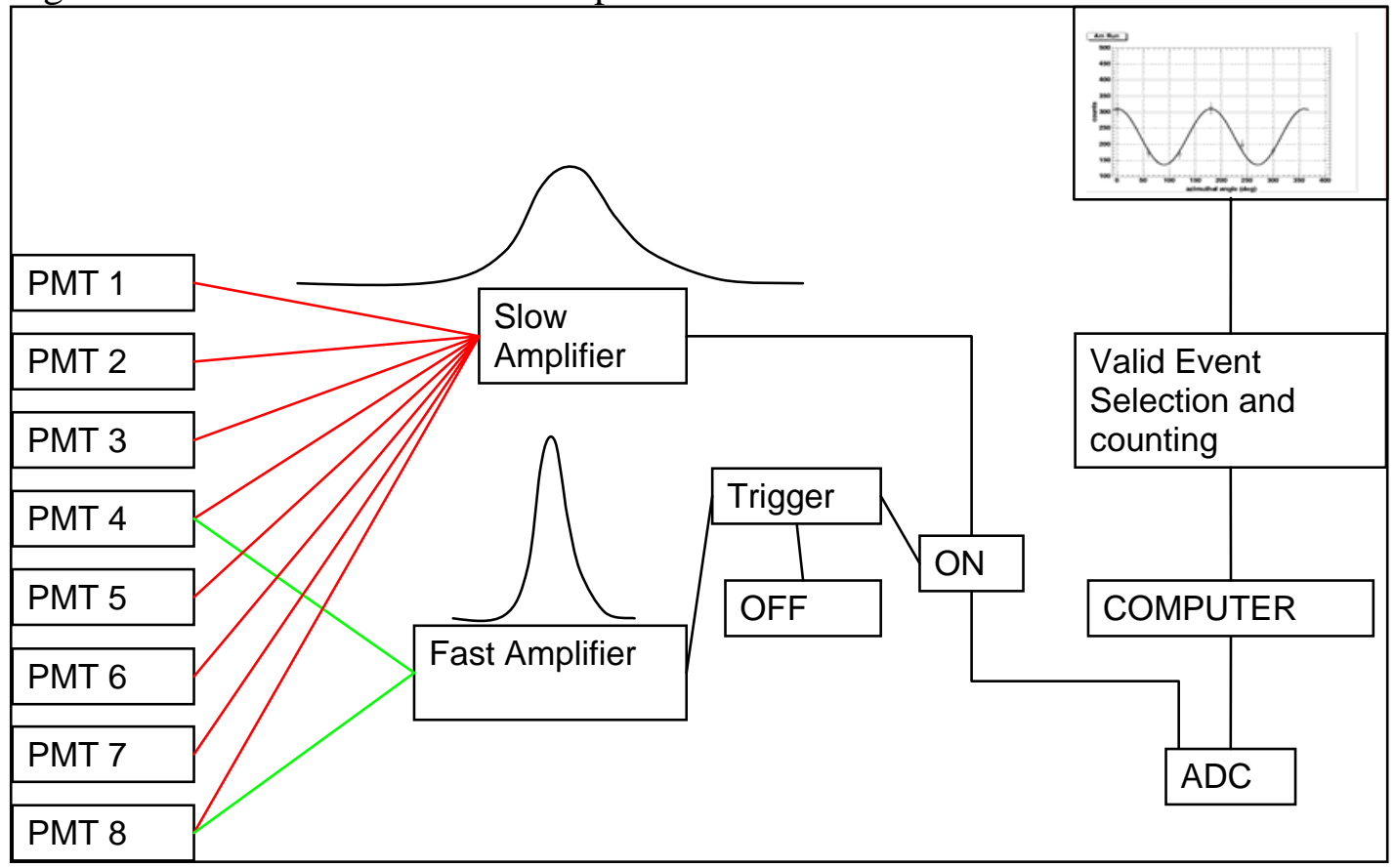

